\title{
The Effect of Previous Wingate Performance Using one Body Region on Subsequent Wingate Performance Using a Different Body Region
}

\author{
by \\ Leonie Harvey ${ }^{1}$, Matthew Bousson ${ }^{1}$, Chris McLellan², Dale Lovell ${ }^{1}$
}

The 30 second Wingate Anaerobic Test (WAnT) is the gold standard measure of anaerobic performance. The present investigation aimed to determine if a previous WAnT using one body region significantly affected a subsequent WAnT using a different body region. Twelve male university students $(n=12,23 \pm 2$ years, $84 \pm 16.1 \mathrm{~kg}, 178.5 \pm 7.4$ $\mathrm{cm}$ ) volunteered to complete two repeated WAnT protocols (either lower body WAnT followed by an upper body WAnT or vice versa) on two separate testing occasions. The upper body WAnT was conducted on a modified electromagnetically braked cycle ergometer using a flywheel braking force corresponding to 5\% bodyweight. The lower body WAnT was conducted on an electronically braked cycle ergometer using a flywheel braking force corresponding to 7.5\% bodyweight. Participants had a 1 minute rest period for transition between WAnTs. Data are reported as mean \pm standard deviation. No significant differences were identified in power indices for the lower body between $30 \mathrm{~s}$ WAnTs. When the upper body WAnT was performed $2 n d$, absolute peak power $(p<0.01)$, mean power $(p<0.001)$ and relative mean power $(p<0.001)$ were significantly lower compared to when the upper body WAnT was performed $1 s t$. The value of maximum revolutions per minute was significantly lower $(p<0.001)$ when the upper body WAnT was performed after the lower body WAnT, compared to when it was performed 1st (193.3 \pm 11.41 st vs $179.8 \pm 14.42$ nd). Previous upper body sprint exercise does not significantly affect lower body sprint exercise; however, previous lower body sprint exercise severely compromises subsequent upper body sprint performance.

Key words: repeated, WAnT, upper body, lower body.

\section{Introduction}

The 30 second Wingate anaerobic test $(30$ s WAnT) is one of the most commonly implemented tests for measuring anaerobic performance, as it has been shown to be both a valid and reliable tool in the assessment of anaerobic performance in both athletic (Hawley and Williams, 1991; Horswill et al., 1989; Kounalakis et al., 2009) and clinical populations (Jacobs et al., 2003; Metter et al., 2004). This supramaximal intensity test involves $30 \mathrm{~s}$ of all out cycling using either the arms or the legs, both types of this test are performed in a seated position to minimise the contribution of other untargeted musculature and the momentum which may be generated by the individual's body weight (McLester et al., 2004; Reiser et al., 2002). While the upper and lower body $30 \mathrm{~s}$ WAnTs have been extensively researched within the current literature (Beneke et al., 2002; Lovell et al., 2013; Richmond et al., 2011), alternating upper and lower body repeated WAnT performance is yet to be examined.

Examining repeated $30 \mathrm{~s}$ WAnT performance is important as many team and

\footnotetext{
1 - School of Health and Sport Sciences, Faculty of Science, Health E Education, University of the Sunshine Coast, Sippy Downs, Queensland, Australia.

2- Faculty of Health Sciences and Medicine, Bond University, Robina, Queensland, Australia.
} 
individual sports such as rugby league (Lovell et al., 2013), wrestling (Horswill et al., 1992), crosscountry skiing (Mahood et al., 2001; Van Hall et al., 2003) and rock climbing (Bertuzzi et al., 2007; Sheel et al., 2003) require simultaneous or consecutive short duration, explosive efforts from both the upper and lower body. For example, while rugby league appears to be a running based sport, it has been found that an elite rugby league player may make more than 41 tackles per game (Sirotic et al., 2011) with high intensity grappling efforts lasting as long as $35 \mathrm{~s}$ (Gabbett, 2008), a similar time taken as the WAnT. Similarly, during cross-country skiing it has been reported that more than $50 \%$ of forward propulsion is generated by the upper body (Smith et al., 1989), while the relative arm intensity during continuous ski exercise is equal to approximately $60 \%$ of maximal arm VO2 (Van Hall et al., 2003). These statistics relate only to the upper body efforts required by these sports, overlooking the effect and contribution of any lower body efforts. Consequently, the role of different body regions on subsequent high intensity efforts remains unclear.

Although previous investigations have examined repeated $30 \mathrm{~s}$ WAnTs, they included 2 or more WAnTs performed by the same body region, ranging from 10 to $30 \mathrm{~s}$ in duration and separated by 1.5 up to $6 \mathrm{~min}$ of recovery (Bogdanis et al., 1994, 1996; Kohler et al., 2010; Watt et al., 2002). In one of the first investigations to examine repeated lower body 30s WAnT performance, it was reported that peak power (PP) and mean power (MP) output decreased by approximately $20 \%$ in the subsequent lower body 30 s WAnT (Bogdanis et al., 1996). Furthermore, a shift in the energy metabolism was also reported with a $45 \%$ decrease in glycolysis from the first lower body WAnT to the second lower body WAnT, with a concomitant increase of approximately $20 \%$ in aerobic energy contribution (Bogdanis et al., 1996). While it is clear that repeated lower body $30 \mathrm{~s}$ WAnTs have a profound effect on performance, less clear is the effect of upper body $30 \mathrm{~s}$ WAnT performance on subsequent lower body $30 \mathrm{~s}$ WAnT performance or the effect of lower body $30 \mathrm{~s}$ WAnT performance on subsequent upper body $30 \mathrm{~s}$ WAnT performance.

Significant contribution of both the upper and lower body is a requirement in the successful performance of many sports and in turn warrants the division and further investigation into individual components. Therefore, the present investigation aimed to examine the effect of short duration, high intensity exercise using one body region on the subsequent performance of short duration, high intensity exercise using another body region. This was achieved by implementing a randomized crossover design using an upper body $30 \mathrm{~s}$ WAnT and lower body $30 \mathrm{~s}$ WAnT, with $1 \mathrm{~min}$ recovery between efforts. It was hypothesized that subsequent short duration, high intensity upper body exercise would be significantly reduced following a prior short duration, high intensity lower body effort.

\section{Material and Methods}

\section{Participants}

Twelve male university students ( $\mathrm{n}=12$, $23 \pm 2$ years, $84 \pm 16.1 \mathrm{~kg}, 178.5 \pm 7.4 \mathrm{~cm}$ ) volunteered to participate in the study. The subjects were not highly trained in any specific sport, but participated in physical activity, completing $\leq 3$ activity sessions $\cdot \mathrm{wk}^{-1}$. Physical activity included walking, jogging, tennis and recreational sports such as touch football and surfing.

\section{Testing Sessions}

All participants reported to the laboratory on three different occasions which were separated by a minimum of 5 days. During the first visit, participants completed a medical history questionnaire and the required pre-screening procedures. It allowed us to state that all participants were healthy and free from any cardiovascular or neuromuscular irregularities. Upon the fulfilment of this inclusion criteria, participants were then familiarized with the electro-magnetically braked cycle ergometer (Excalibur Sport, Lode B.V., Netherlands) which was used for upper body testing and the electronically braked cycle ergometer (Velotron, Racermate, Inc., Seattle, USA) used for lower body testing, in addition to the Repeated $30 \mathrm{~s}$ Wingate Anaerobic Test (WAnT) protocol.

During the second visit, participants completed one Repeated $30 \mathrm{~s}$ WAnT (either lower body $30 \mathrm{~s}$ WAnT followed by an upper body $30 \mathrm{~s}$ WAnT or vice versa). During the final visit, participants completed the remaining Repeated 30 
s WAnT. Testing order was randomized to ensure no learning effect occurred. Prior to the commencement of the study, the experimental procedures and potential risks were explained to the participants and all provided written informed consent. All relevant research ethic applications had been approved by the University of the Sunshine Coast Ethics Committee, application reference number (S/09/233), and performed in accordance with the Declaration of Helsinki.

\section{Repeated WAnT protocol}

The upper body $30 \mathrm{~s}$ WAnT was conducted on a modified electro-magnetically braked cycle ergometer (EE) (Excalibur Sport, Lode B.V., Netherlands). The EE was fixed to a table, with the table fixed to the ground to prevent any movement in the EE during the WAnT (Lovell et al., 2011). Participants sat on a chair (also fixed to the ground) then they were advised to keep their feet flat on the ground and remain seated throughout the WAnT. The seat height and back rest were adjusted so that with the crank position on the opposite side to the body and the hand grasping the handles, the elbow joint was almost in full extension $\left(165-175^{\circ}\right)$ and the shoulders in line with the center of the ergometers shaft.

The lower body 30 s WAnT was conducted on an electronically braked cycle ergometer (Velotron, Racermate, Inc., Seattle, USA). The seat height was adjusted so that when the pedal was positioned at the lowest point, the knee joint was almost in full extension (approximately $5^{\circ}$ of flexion). Participants were instructed to grasp the handlebars and remain seated for the duration of the test. A fly wheel braking force corresponding to $5 \%$ and $7.5 \%$ of the participants body weight was used for the upper and lower body, respectively (Dotan and Bar-Or, 1983; Inbar et al., 1996).

Prior to the commencement of the Repeated WAnT, participants completed a $5 \mathrm{~min}$ warm-up at 50W which included three short sprint efforts followed by $5 \mathrm{~min}$ recovery. Then the participants stretched for approximately $3 \mathrm{~min}$ before the beginning of the test. Participants were instructed to arm crank/cycle as fast as possible and were given a $3 \mathrm{~s}$ countdown before the set resistance was applied. Verbal encouragement was given to all participants to maintain their highest possible cadence throughout both WAnTs. Upon completing the first WAnT, participants had a 1 min rest period for transition to the next piece of testing equipment to perform the remaining $30 \mathrm{~s}$ WAnT. A time frame of $1 \mathrm{~min}$ was selected to ensure adequate time for safe transition to the next piece of testing equipment and to be fully set up, while not allowing so much rest that the subsequent $30 \mathrm{~s}$ WAnT would be favourably affected.

Power output was recorded by the Wingate version 1.0.7 software (Lode B.V., Netherlands) and Velotron CS software (RacerMate Inc., Seattle, USA) for the upper and lower body WAnT, respectively. The following WAnT variables were measured: Peak power (PP) was calculated as the highest single point of power output (recorded at $0.2 \mathrm{~s}$ intervals). Mean power (MP) was the average power output during the $30 \mathrm{~s}$ test. The fatigue index (FI) was reported as a single value that was calculated using Wingate version 1.0.7 software (Lode B.V., Netherlands) and Velotron CS software (RacerMate Inc., Seattle, USA).

\section{Statistical Analysis}

All analyses were performed using the IBM SPSS 21.0 program for Windows (Chicago, IL). Data are reported as means \pm standard deviation (SD). An independent t-test was used to determine significant differences in performance measures for the repeated $30 \mathrm{~s}$ WAnT within the one body region (eg Arms $1^{\text {st }}$ vs Arms $2^{\text {nd }}$ ). Performance measures included: absolute peak power (PP) and mean power (MP) measured in Watts $(\mathrm{W})$, relative PP and MP measured in Watts per kilogram of bodyweight $\left(\mathrm{W} \cdot \mathrm{kg}^{-1}\right)$, a fatigue index (FI) and maximum revolutions per minute (Max RPM). The level of significance of $5 \%(p<$ 0.05 ) was adopted in all analyses.

\section{Results}

Participants were all healthy, physically active males of a similar age. These selection criteria ensured a homogenous participant group and minimized the effect of any potentially confounding variables. Performance data from the repeated $30 \mathrm{~s}$ WAnTs are displayed in Table 1. Both absolute (W) and relative $\left(\mathrm{W} \cdot \mathrm{kg}^{-1}\right) \mathrm{PP}$ and MP did not differ significantly for the lower body $30 \mathrm{~s}$ WAnT when performed first, compared to when it was performed $2^{\text {nd }}$ (following the upper 
body $30 \mathrm{~s}$ WAnT). Conversely, when the upper body $30 \mathrm{~s}$ WAnT was performed $2^{\text {nd }}$ (following a lower body $30 \mathrm{~s}$ WAnT), absolute PP $(p<0.01)$, MP $(p<0.001)$ and relative MP $(p<0.001)$ were significantly lower compared to when the upper body $30 \mathrm{~s}$ WAnT was performed first. The FI for both the arms and legs did not differ significantly between the $1^{\text {st }}$ and $2^{\text {nd }} 30 \mathrm{~s}$ WAnT efforts. The maximum RPM measured for the legs was not significantly different between the $1^{\text {st }}$ lower body $30 \mathrm{~s}$ WAnT effort compared to when it was performed $2^{\text {nd }}$ (following the upper body $30 \mathrm{~s}$ WAnT). The maximum RPM measured for the arms was significantly lower $(p<0.001)$ when the upper body $30 \mathrm{~s}$ WAnT was performed after the lower body $30 \mathrm{~s}$ WAnT, compared to when it was performed $1^{\text {st }}\left(193.3 \pm 11.41^{\text {st }}\right.$ vs $\left.179.8 \pm 14.42^{\text {nd }}\right)$.

Table 1

Performance measures for the Repeated $30 \mathrm{~s}$ WAnT protocols for the arms and legs $(N=12)$. NB: Comparisons are made within one body region (eg Arms $1^{\text {st }}$ vs Arms $2^{\text {nd }}$ ).

\begin{tabular}{lcccc}
\hline & \multicolumn{2}{c}{ WAnT A } & \multicolumn{2}{c}{ WAnT B } \\
Measure & Arms $\left(1^{\text {st }}\right)$ & Legs $\left(2^{\text {nd }}\right)$ & Legs $\left(1^{\text {st }}\right)$ & Arms $\left(2^{\text {nd }}\right)$ \\
\hline PP $(\mathrm{W})$ & $753.7 \pm 150.2$ & $935.8 \pm 205.5$ & $931 \pm 198.2$ & $706.3 \pm 143.4^{\mathrm{a}}$ \\
MP $(\mathrm{W})$ & $568.6 \pm 117.4$ & $564.7 \pm 98.7$ & $573.2 \pm 100.8$ & $484.4 \pm 90.1^{\mathrm{b}}$ \\
PP $\left(\mathrm{W} \cdot \mathrm{kg}^{-1}\right)$ & $7.6 \pm 3.6$ & $11.1 \pm 1.1$ & $11.1 \pm 1.5$ & $8.4 \pm 0.9$ \\
MP $\left(\mathrm{W} \cdot \mathrm{kg}^{-1}\right)$ & $6.8 \pm 0.3$ & $6.7 \pm 0.4$ & $6.8 \pm 0.4$ & $5.8 \pm 0.6^{\mathrm{b}}$ \\
FI & $45.6 \pm 12.2$ & $22.3 \pm 5.8$ & $22.6 \pm 7.4$ & $43.2 \pm 11.1$ \\
Max RPM & $193.3 \pm 11.4$ & $180.1 \pm 10.3$ & $176.3 \pm 10.5$ & $179.8 \pm 14.4^{\mathrm{b}}$ \\
\hline
\end{tabular}

$P P$, peak power; $M P$, mean power; $(W)$, watts; $\left(W \cdot \mathrm{kg}^{-1}\right)$, watts per kilogram;

FI, fatigue index; Max RPM, maximum revolutions per minute;

Data is displayed as the mean $\pm S D$, standard deviation: ${ }^{a} p<0.01$ from when performed $1^{\text {st }} ;{ }^{b} p<0.001$ from when performed $1^{\text {st; }}$;

\section{Discussion}

The main finding of the present investigation was that lower body $30 \mathrm{~s}$ WAnT performance was not significantly affected by a previous upper body $30 \mathrm{~s}$ WAnT performance. Conversely, the upper body $30 \mathrm{~s}$ WAnT was significantly reduced following a lower body $30 \mathrm{~s}$ WAnT, confirming our hypothesis. Furthermore, while previous exercise did not significantly affect the maximum RPM achieved during the lower body $30 \mathrm{~s}$ WAnT, the maximum RPM for the upper body $30 \mathrm{~s}$ WAnT was significantly reduced following prior leg exercise.

To the best of our knowledge, no previous investigations have examined the effect of preceding, short duration, high intensity sprint efforts on subsequent sprint efforts performed by a different region of the body. The present investigation found that both absolute $(\mathrm{W})$ and relative $(\mathrm{W} \bullet \mathrm{kg}-1) \mathrm{PP}$ output was not significantly different between lower body $30 \mathrm{~s}$ WAnT efforts (Table 1), suggesting that prior upper body $30 \mathrm{~s}$ 
WAnT performance did not significantly impact power output during the lower body $30 \mathrm{~s}$ WAnT. Alternatively, it was found that absolute (W) PP output for the upper body $30 \mathrm{~s}$ WAnT was significantly $(p<0.01)$ reduced following a lower body $30 \mathrm{~s}$ WAnT, indicating that the upper body $30 \mathrm{~s}$ WAnT was significantly affected by previous lower body exercise. These findings are in agreement with some (Bogdanis, 2012; Bogdanis et al., 1994), but not others (Halperin et al., 2014) who have attempted to examine the effect of high intensity exercise targeting one body region on another body region. In a previous investigation, 5 min of heavy arm cranking at $30 \%$ of mean power output was performed prior to a lower body $30 \mathrm{~s}$ WAnT. It was reported that despite the arm cranking exercise elevating blood lactate (BLaᄀ-) levels to $11.0 \pm 0.6 \mathrm{mM}$, no significant change in PP and MP was observed in the subsequent lower body $30 \mathrm{~s}$ WAnT (Bogdanis et al., 1994). Conversely, Halperin et al. (2014) found that elbow flexor force was significantly $(p<0.05)$ decreased following high intensity exercise of the knee extensor musculature. However, the relevance of the methodologies used in the above mentioned studies and their application to high intensity, intermittent exercise is questionable. The present investigation utilized repeated, maximal effort Wingate exercise using different body regions to more closely resemble high intensity sporting performance.

During the $30 \mathrm{~s}$ WAnT, PP output is indicative of instantaneous force output which relies on intramuscular stores of adenosine triphosphate (ATP) and phosphocreatine (PCr) (Baker et al., 2010). While the present investigation found that the lower body $30 \mathrm{~s}$ WAnT performance was not affected by prior upper body exercise, the upper body $30 \mathrm{~s}$ WAnT performance was significantly affected by prior lower body exercise. A possible explanation for these findings may be that upper body intramuscular ATP/PCr stores were used during the lower body $30 \mathrm{~s}$ WAnT as a result of gripping the handlebars (Baker et al., 2001). Consequently, the $1 \mathrm{~min}$ transition period between the lower and upper body $30 \mathrm{~s}$ WAnT testing equipment in the present investigation may not have been sufficient enough to allow the full replenishment of the upper body intramuscular ATP/PCr stores in preparation for the subsequent upper body $30 \mathrm{~s}$
WAnT (Bogdanis et al., 1995).

The present investigation also found that both absolute $(\mathrm{W})$ and relative $(\mathrm{W} \bullet \mathrm{kg}-1) \mathrm{MP}$ output was significantly $(\mathrm{p}<0.01)$ lower for the upper body $30 \mathrm{~s}$ WAnT when it was performed following a lower body $30 \mathrm{~s}$ WAnT. A plausible explanation for these findings may be attributed to the difference in energy system contributions between the upper and lower body $30 \mathrm{~s}$ WAnT. While the present investigation did not directly examine energy system contributions during the WAnTs, our laboratory had previously found that the anaerobic lactic energy system contributed over $60 \%$ of the energy requirements during the upper body $30 \mathrm{~s}$ WAnT, compared to less than $50 \%$ of the energy requirements during the lower body 30 s WAnT (Harvey et al., 2015). Similar contributions have been reported elsewhere for both the upper (Lovell et al., 2013) and lower body (Beneke et al., 2002; Granier et al., 1995; Harvey et al., 2015) WAnTs. Consequently the lower MP output observed during the subsequent upper body WAnT may be attributed to the increase in $\mathrm{H}+$ concentration from the lower body WAnT, impairing the glycolytic (anaerobic lactic) energy production for the subsequent upper body WAnT (Bogdanis et al., 1995). Furthermore, the gripping action experienced during the upper body WAnT may have occluded the blood flow, leading to a higher local $\mathrm{H}+$ concentration and a subsequent decrease in MP output (Bogdanis et al., 1995). However, during the lower body WAnT the cyclical motion may have allowed for a greater blood flow and therefore increased $\mathrm{H}+$ clearance and oxygen supply (Spriet et al., 1989). Consequently, no significant difference was found in MP output when the lower body WAnT was performed following the upper body WAnT. Additionally, the higher aerobic contribution of the lower body WAnT performance may have also reduced the reliance on the lower body glycolytic energy production and therefore, fatigue (Harvey et al., 2015).

As with all WAnTs, the resistance remains fixed for the duration of the sprint, therefore reductions in power are a result of changes in the number of revolutions performed. The results of the present investigation indicated that the RPMs for the upper body were significantly reduced following the lower body WAnT, while no significant differences were found between lower 
body WAnT efforts. These changes may have occurred as a result of peripheral musculature fatigue in the upper body and the inability to regenerate ATP due to the reasons mentioned above. More recent evidence also indicates that the central nervous system may regulate muscle recruitment and limit performance during fatigue in short intermittent sprints (Billaut et al., 2013).

While this investigation provides the first examination of back-to-back WAnTs using different body regions, there were some limitations to be noted. The present investigation examined back-to-back WAnT performance in physically active males only. Although this provided important information, future investigations should examine this protocol with females due to significant differences in gender responses to high intensity exercise (Ben Ari et al., 1978; Murphy et al., 1984). Furthermore, athletes (both male and female) that are involved in sports requiring both upper and lower body performance should be examined to determine the effect of long term training on the physiological responses to back-to-back WAnT. Another limitation of this investigation is the lack of assessment of biochemical variables which may help to explain the results obtained. The present investigation did not examine the effect of two consecutive bouts of short duration, high intensity exercise on blood lactate or hydrogen ion $(\mathrm{H}+)$ concentrations which would provide an insight into the physiological state of the body after the upper and lower body exercise efforts. As such, future studies should measure these blood markers pre and post back-to-back exercise to provide a better understanding of the physiological strain from one body region and its effect on subsequent performance from another body region. Heart rate and oxygen debt variables should also be assessed and used to supplement biochemical data.

In summary, the present investigation found that while previous upper body sprint exercise did not significantly affect lower body sprint exercise, previous lower body sprint exercise severely compromised subsequent upper body sprint performance. Furthermore, the maximum RPM for the upper body $30 \mathrm{~s}$ WAnT was significantly reduced following prior lower body sprint exercise. These findings have noteworthy consequences for sporting performance that involves both upper and lower body high intensity efforts. As such, future investigations should aim to further examine the mechanisms which cause fatigue in the upper and lower body musculature to help better understand why prior lower body exercise significantly impairs subsequent body exercise. Additionally, future investigations should also examine intermittent upper and lower body exercise to determine if similar results are found.

\section{Practical Implications}

- Training adaptations are dependent upon the specific type of training that is practiced. Therefore, athletes that participate in sports which require power and anaerobic capacity from both the upper and lower limbs should be mindful of the order of activities performed during training, as exercise order will significantly affect performance and ultimately the promotion of favourable training adaptations.

- Lower body high intensity exercise performance is not significantly affected by previous upper body efforts, therefore lower body testing and training can be performed before or after upper body training at no detriment to lower body power output.

- When looking to increase or assess upper body power and anaerobic capacity, coaches should be aware that upper body high intensity exercise performance will be significantly reduced following high intensity lower body efforts and should therefore be sequenced appropriately throughout training sessions.

\section{Acknowledgements}

We would like to thank and acknowledge the University of the Sunshine Coast for providing all testing facilities and also the participants for volunteering their time. This manuscript represents original work that is not being considered for publication, in whole or in part, in another journal, book, conference proceedings, or government publication with a substantial circulation. All previously published work cited in the manuscript has been fully acknowledged. 


\section{References}

Baker J, Gal J, Davies B, Bailey D, Morgan R. Power output of legs during high intensity cycle ergometry: Influence of hand grip. J Sci Med Sport, 2001; 4: 10-18

Baker JS, McCormick MC, Robergs RA. Interaction among Skeletal Muscle Metabolic Energy Systems during Intense Exercise. J Nutr Metab, 2010; 2010: 905612

Ben Ari E, Inbar O, Bar-Or O. The aerobic capacity and maximal anaerobic power of 30-to-40-year-old men and women. In Book G, Landry F, Orban W, editors. Proceedings of the International Congress of Physical Activity Sciences. Quebec; International Congress of Physical Activity Sciences, 427-433; 1978

Beneke R, Pollmann C, Bleif I, Leithauser RM, Hutler M. How anaerobic is the Wingate Anaerobic Test for humans? Eur J Appl Physiol, 2002; 87: 388-392

Bertuzzi RC, Franchini E, Kokubun E, Kiss MA. Energy system contributions in indoor rock climbing. Eur J Appl Physiol, 2007; 101: 293-300

Billaut F, Kerris JP, Rodriguez RF, Martin DT, Gore CJ, Bishop DJ. Interaction of central and peripheral factors during repeated sprints at different levels of arterial O2 saturation. PloS one, 2013; 8: e77297

Bogdanis GC. Effects of physical activity and inactivity on muscle fatigue. Front Physiol, 2012; 3: 142

Bogdanis GC, Nevill ME, Boobis LH, Lakomy H. Contribution of phosphocreatine and aerobic metabolism to energy supply during repeated sprint exercise. J Appl Physiol, 1996; 80: 876-884

Bogdanis GC, Nevill ME, Boobis LH, Lakomy H, Nevill AM. Recovery of power output and muscle metabolites following $30 \mathrm{~s}$ of maximal sprint cycling in man. J Physiol, 1995; 482: 467

Bogdanis GC, Nevill ME, Lakomy HK. Effects of previous dynamic arm exercise on power output during repeated maximal sprint cycling. J Sports Sci, 1994; 12: 363-370

Dotan R, Bar-Or O. Load optimization for the Wingate Anaerobic Test. Eur J Appl Physiol Occup Physiol, 1983; 51: $409-417$

Gabbett TJ. Influence of fatigue on tackling technique in rugby league players. J Strength Cond Res, 2008; 22: 625-632

Granier P, Mercier B, Mercier J, Anselme F, Préfaut C. Aerobic and anaerobic contribution to Wingate test performance in sprint and middle-distance runners. Eur J Appl Physiol, 1995; 70: 58-65

Halperin I, Aboodarda SJ, Behm DG. Knee extension fatigue attenuates repeated force production of the elbow flexors. Eur J Sport Sci, 2014; 14: 823-829

Harvey L, Wiegand A, Solomon C, McLellan C, Lovell D. A comparison of upper and lower body energetics during high-intensity exercise. J Sport Med Phys Fitness, 2015; 55: 708-713

Hawley JA, Williams MM. Relationship between Upper-Body Anaerobic Power and Freestyle Swimming Performance. Int J Sports Med, 1991; 12: 1-5

Horswill C, Miller J, Scott J, Smith C, Welk G, Van Handel P. Anaerobic and aerobic power in arms and legs of elite senior wrestlers. Int J Sports Med, 1992; 13: 558-561

Horswill CA, Scott JR, Galea P. Comparison of maximum aerobic power, maximum anaerobic power, and skinfold thickness of elite and nonelite junior wrestlers. Int J Sports Med, 1989; 10: 165-168

Inbar O, Bar-Or O, Skinner JS. The Wingate Anaerobic Test. Champaign, IL: Human Kinetics; 1996

Jacobs PL, Mahoney ET, Johnson B. Reliability of arm Wingate Anaerobic Testing in persons with complete paraplegia. J Spinal Cord Med, 2003; 26: 141-144

Kohler RM, Rundell KW, Evans TM, Levine AM. Peak power during repeated wingate trials: implications for testing. J Strength Cond Res, 2010; 24: 370-374

Kounalakis SN, Koskolou MD, Geladas ND. Oxygen saturation in the triceps brachii muscle during an arm Wingate test: the role of training and power output. Res Sports Med, 2009; 17: 171-181 
Lovell D, Kerr A, Wiegand A, Solomon C, Harvey L, McLellan C. The contribution of energy systems during the upper body Wingate anaerobic test. Appl Physiol Nutr Metab, 2013; 38: 216-219

Lovell D, Mason D, Delphinus E, Eagles A, Shewring S, McLellan C. Does upper body strength and power influence upper body Wingate performance in men and women? Int J Sports Med, 2011; 32: 771-775

Lovell D, Mason D, Delphinus E, McLellan C. Upper and lower body anaerobic performance of semi-elite Rugby League players. J Sports Med Phys Fitness, 2013; 53: 477-482

Mahood NV, Kenefick RW, Kertzer R, Quinn TJ. Physiological determinants of cross-country ski racing performance. Med Sci Sports Exerc, 2001; 33: 1379-1384

McLester JR, Green JM, Chouinard JL. Effects of standing vs. seated posture on repeated Wingate performance. J Strength Cond Res, 2004; 18: 816-820

Metter EJ, Talbot LA, Schrager M, Conwit RA. Arm-cranking muscle power and arm isometric muscle strength are independent predictors of all-cause mortality in men. J Appl Physiol, 2004; 96: 814-821

Murphy MM, Patton JF, Frederick FA. A comparison of anaerobic power capacity in males and females accounting for differences in thigh volume, body weight and lean body mass. Med Sci Sports Exerc, 1984; 16: 108

Reiser RF, Maines JM, Eisenmann JC, Wilkinson JG. Standing and seated Wingate protocols in human cycling. A comparison of standard parameters. Eur J Appl Physiol, 2002; 88: 152-157

Richmond SR, Whitman SA, Acree LS, Olson BD, Carper MJ, Godard MP. Power output in trained male and female cyclists during the Wingate test with increasing flywheel resistance. J Exerc Physiol Online, 2011; 14: 46-53

Sheel AW, Seddon N, Knight A, McKenzie DC. Physiological responses to indoor rock-climbing and their relationship to maximal cycle ergometry. Med Sci Sports Exerc, 2003; 35: 1225-1231

Sirotic AC, Knowles H, Catterick C, Coutts AJ. Positional match demands of professional rugby league competition. J Strength Cond Res, 2011; 25: 3076-3087

Smith GA, Nelson RC, Feldman A, Rankinen JL. Analysis of V1 skating technique of Olympic cross-country skiers. Int J Sport Biomech, 1989; 5: 185-207

Spriet L, Lindinger M, McKelvie R, Heigenhauser G, Jones N. Muscle glycogenolysis and H+ concentration during maximal intermittent cycling. J Appl Physiol, 1989; 66: 8-13

Van Hall G, Jensen-Urstad M, Rosdahl H, Holmberg H-C, Saltin B, Calbet JA. Leg and arm lactate and substrate kinetics during exercise. Am J Physiol Endocrinol Metab, 2003; 284: E193-E205

Watt KKO, Hopkins WG, Snow RJ. Reliability of performance in repeated sprint cycling tests. J Sci Med Sport, 2002; 5: 354-361

\section{Corresponding author:}

\section{Leonie Harvey}

School of Health and Sport Sciences, Faculty of Science, Health \& Education, University of the Sunshine

Coast, 90 Sippy Downs Drive, Sippy Downs, Queensland, Australia 4556

Telephone: 61488191276

Facsimile: 61754304880

Email:1mh009@student.usc.edu.au 